\title{
Special Issue "Selected Papers from the 2019 42nd International Conference on Telecommunications and Signal Processing (TSP)"
}

\author{
Norbert Herencsar ${ }^{1, *(1)}$, Francesco Benedetto ${ }^{2}(\mathbb{D})$ and Jorge Crichigno ${ }^{3}(\mathbb{C})$ \\ 1 Department of Telecommunications, Brno University of Technology, Technicka 3082/12, \\ 61600 Brno, Czech Republic \\ 2 Signal Processing for Telecommunications and Economics Laboratory (SP4TE), University of "Roma TRE", \\ via Vito Volterra 62, 00146 Rome, Italy; francesco.benedetto@uniroma3.it \\ 3 College of Engineering and Computing, University of South Carolina, Innovation Center, \\ 550 Assembly Street, Suite 1300, Columbia, SC 29208, USA; jcrichigno@cec.sc.edu \\ * Correspondence: herencsn@feec.vutbr.cz
}

Received: 25 February 2020; Accepted: 13 March 2020; Published: 19 March 2020

\section{Dear Readers,}

This Special Issue contains a series of excellent research works on telecommunications and signal processing; selected from the 2019 42nd International Conference on Telecommunications and Signal Processing (TSP), which was held during 1-3 July 2019, in Budapest, Hungary. The Conference was organized in cooperation with the IEEE Region 8 (Europe, Middle East and Africa), IEEE Hungary Section, IEEE Czechoslovakia Section, IEEE Czechoslovakia Section SP/CAS/COM Joint Chapter, and Scientific Association for Infocommunications, a Sister Society of the IEEE and the IEEE Communications Society, by seventeen universities from Czech Republic, Hungary, Turkey, Taiwan, Japan, Slovak Republic, Spain, Bulgaria, France, Slovenia, Croatia, Greece, and Poland, for academics, researchers, and developers, and it serves as a premier annual international forum to promote the exchange of the latest advances in telecommunication technology and signal processing [1]. The aim of the conference is to bring together both novice and experienced scientists, developers, and specialists, to meet new colleagues, collect new ideas, and establish new cooperation between research groups from universities, research centers, and private sectors worldwide. It is our great pleasure to introduce a collection of 11 selected high-quality research papers and let us briefly illustrate the published works in this Special Issue.

The deployment of new radio access technologies always provides a good opportunity and timing to optimize the existing mobile front- and backhaul (commonly called "anyhaul"). The legacy systems (Long-Term Evolution (LTE), High-Speed Packet Access (HSPA), third-generation mobile (3G), second-generation mobile (2G)) already extensively utilize the transmission and transport capacities of the mobile anyhaul. With the current launch of 5G (fifth-generation mobile) and recent LTE-A (Advanced Long-Term Evolution), additional new transmission capacities are required again. Depending on the traffic and network topology, additional cell sites are built, and even more locations are connected with fiber optics. The existing microwave and millimeter-wave links are rotated toward those aggregation points that already have optical-fiber access. Due to the increased cell-site density, the average distance of the radio access links can be reduced by network and topology optimization. The reduced hop lengths combined with adaptive modulation and automatic power control bring an opportunity for capacity increase in shortened radio links. Links newly deployed for 5G find a wide spectrum in the millimetric V, E, W, and D frequency bands. In the first paper of this Special Issue [2], written by A. Hilt, the author discusses the availability and hop-length targets of the anyhaul links that should be carefully kept by proper planning and monitoring.

Passive optical networks are currently the most promising solution for access networks. These networks rely on broadcast signal distribution in the downstream direction and unicast signal 
transmission in the upstream direction. The upstream direction is controlled by optical line termination (OLT). The broadcast transmission method increases security vulnerability because the attacker is able to connect his/her modified optical network unit (ONU) to the free port of the splitter (commonly in the basement). In the next paper [3], V. Clupek et al. present the concept for the activation process of ONUs based on physical unclonable function (PUF) for next-generation passive optical networks stage 2 (NG-PON2). The use of PUF increases security in the NG-PON2. Furthermore, the registration identifier (ID) is not stored in a nonvolatile memory, in comparison with the common solution defined by the International Telecommunication Union (ITU) recommendation G.989.3. An attacker cannot perform a reverse engineering attack to obtain the registration ID. For this reason, the attacker cannot clone an ONU. Authors proposed security improvements that involve authentication, encryption, integrity protection, and data origin verification methods in the NG-PON2. The proposed model uses the standard implementation of the transmission convergence layer of NG-PON2 with the new physical layer operations, administration, and maintenance (PLOAM) messages. The recommendation G.989.3 allows specifying own PLOAM messages since not all IDs are used in the current specification.

In today's age of digital media and social networks, delivering content to a massive scale of recipients became one of the main challenges. Load Balancers and Content Delivery Networks are used to distribute content to multiple locations, which increase the scalability of services and decrease the load on content origins. Both technologies rely on redirections. Redirections have not received a significant amount of attention in the recent years; however, they do impose some limitations. In the article [4], T. Boros et al. propose a transparent redirection mechanism, which exploits the versatility of Software Defined Networking. The redirection method is achieved by handing off existing TCP sessions without any required modifications to the endpoints. This article demonstrates how the proposed redirection mechanism can be adopted in Content Delivery Networks and Load Balancing scenarios. The performance of the solution is thoroughly tested and compared to existing legacy solutions.

The paper [5], written by R. Sotner et al., targets the design and analysis of specific types of transfer functions obtained by the summing operation of integer-order and fractional-order two-port responses. Various operations provided by fractional-order, two-terminal devices have been studied recently. However, this topic needs to be further studied, and the topologies need to be analyzed in order to extend the state of the art. The studied topology utilizes the passive solution of a constant-phase element (with order equal to 0.5 ) implemented by parallel resistor-capacitor circuit (RC) sections operating as a fractional-order two-port. The integer-order part is implemented by operational amplifier-based lossless integrators and differentiators in branches with electronically adjustable gain, useful for time constant tuning. Four possible cases of the fractional-order and integer-order two-port interconnections are analyzed analytically, by PSpice simulations and also experimentally in the frequency range between $10 \mathrm{~Hz}$ and $1 \mathrm{MHz}$. Standard discrete active components are used in this design for laboratory verification. Practical recommendations for construction and also particular solutions overcoming possible issues with instability and DC offsets are also given. Experimental and simulated results reflect theoretical calculations.

Beat detection systems are widely used in the music information retrieval (MIR) research field for the computation of tempo and beat time positions in audio signals. One of the most important parts of these systems is usually onset detection. There is an understandable tendency to employ the most accurate onset detector. However, there are options to increase the global tempo (GT) accuracy and also the detection accuracy of beat positions at the expense of less accurate onset detection. The study by M. Istvanek et al. [6] aims to introduce an enhancement of a conventional beat detector. The enhancement is based on the Teager-Kaiser energy operator (TKEO), which pre-processes the input audio signal before the spectral flux calculation. The proposed approach is first evaluated in terms of the ability to estimate the GT and beat positions accuracy of given audio tracks compared to the same conventional system without the proposed enhancement. The accuracy of the GT and average beat differences (ABD) estimation is tested on the manually labelled reference database. Finally, this system is used for analysis of a string quartet music database. Results suggest that the presence of 
the TKEO lowers onset detection accuracy but also increases the GT and ABD estimation. The average deviation from the reference GT in the reference database is $9.99 \mathrm{BPM}(11.28 \%)$, which improves the conventional methodology, where the average deviation is 18.19 BPM $(17.74 \%)$. This study has a pilot character and provides some suggestions for improving the beat tracking system for music analysis.

In the paper [7], G. Ulacha and C. Wernik present the improvement of the cascaded prediction method. Three types of main predictor block with different levels of complexity were compared, including two complex prediction methods with backward adaptation, i.e., extension Active Level Classification Model (ALCM+) and extended Ordinary Least Square (OLS+). Authors' approach to implementation of the effective context-dependent constant component removal block is also presented. Additionally, the improved adaptive arithmetic coder with short, medium and long-term adaptation was presented, and the experiment was carried out comparing the results with other known lossless audio coders against which here proposed method obtained the best efficiency.

The article by O. Datcu et al. [8] presents a configurable, high-throughput pseudo-random number generator template targeting cryptographic applications. The template is parameterized using a chaotic map that generates data, an entropy builder that is used to periodically change the parameters of the map and a parameter change interval, which is the number of iterations after which the entropy builder will change the generator's parameters. The system is implemented in $\mathrm{C}++$ and evaluated using the TestU01 and NIST RNG statistical tests. The same implementation is used for a stream cipher that can encrypt and decrypt PNG images. A Monte-Carlo analysis of the seed space was performed. Results show that for certain combinations of maps and entropy builders, more than $90 \%$ of initial states (seeds) tested pass all statistical randomness tests. Furthermore, the throughput is large enough so that an $8 \mathrm{~K}$ color image can be encrypted in $2 \mathrm{~s}$ on a modern laptop CPU (exact specifications are given in the paper). The conclusion is that chaotic maps can be successfully used as a building block for cryptographic random number generators.

In [9], G. Romano considered the problem of the estimation of signal-to-noise ratio (SNR) with a real deterministic sinusoid with unknown frequency, phase and amplitude in additive Gaussian noise of unknown variance. A blind SNR estimator that does not require the knowledge of the instantaneous frequency of the sinusoid, through separate estimation of signal and noise power, was derived using the method of moments, a general method to derive estimators based on high-order moments. Statistical performances of the proposed estimators were studied theoretically through derivation of Cramer-Rao lower bounds (CRLBs) and asymptotic variances. Furthermore, results from Monte-Carlo simulations that confirm the validity of the theoretical analysis are presented along with some comments on the use of proposed estimators in practical applications.

The subject matter of the research article [10] is automatic detection of pavement distress on highway roads using computer vision algorithms. Specifically, deep learning convolutional neural network models are employed towards the implementation of the detector. Source data for training the detector come in the form of orthoframes acquired by a mobile mapping system. Compared to authors' previous work, the orthoframes are generally of better quality, but more importantly, in this work, A. Riid et al. introduce a manual preprocessing step: sets of orthoframes are carefully selected for training and manually digitized to ensure adequate performance of the detector. Pretrained convolutional neural networks are then fine-tuned for the problem of pavement distress detection. Corresponding experimental results are provided and analyzed and indicate a successful implementation of the detector.

Nonalcoholic fatty liver disease (NAFLD) is responsible for a wide range of pathological disorders. It is characterized by the prevalence of steatosis, which results in excessive accumulation of triglyceride in the liver tissue. At high rates, it can lead to a partial or total occlusion of the organ. In contrast, nonalcoholic steatohepatitis (NASH) is a progressive form of NAFLD, with the inclusion of hepatocellular injury and inflammation histological diseases. Since there is no approved pharmacotherapeutic solution for both conditions, physicians and engineers are constantly in search for fast and accurate diagnostic methods. The proposed work by A. Arjmand et al. [11] introduces a fully automated classification 
approach, taking into consideration the high discrimination capability of four histological tissue alterations. The proposed work utilizes a deep supervised learning method, with a convolutional neural network (CNN) architecture achieving a classification accuracy of 95\%. The classification capability of the new $\mathrm{CNN}$ model is compared with a pre-trained AlexNet model, a visual geometry group (VGG)-16 deep architecture and a conventional multilayer perceptron (MLP) artificial neural network. The results show that the constructed model can achieve better classification accuracy than VGG-16 (94\%) and MLP (90.3\%), while AlexNet emerges as the most efficient classifier (97\%).

Modern approaches to physical therapy often use electric currents induced by time-varying magnetic fields. Although some of these methods are already commonly used, and only a few studies are looking at applying particular techniques on exposed tissue. In the last study of the Special Issue [12], written by L. Hanáková et al., a high-induction magnetic stimulation (HIMS) was applied to the chest area to affect the electrical conduction system of the heart. The animal model Sus scrofa domesticus was used for the study. Standard methods were used to make the subsequent analysis, i.e., heart rate variability in time and frequency domain. Concerning the nonlinear character of the electrocardiographic signal and evaluating complex variability (complexity), recurrent quantification analysis was used. The results show high resistance to a physiologically working heart, but there are also specific changes concerning complex variability. Thus, the results indicate that the HIMS application in the chest area may not pose a significant risk to healthy individuals in terms of the short-term effect of this technique on cardiac activity. However, cardiac activity is still, to some extent, affected by the HIMS application. In view of this and the fact that the study was conducted on an animal model, further research in this area would be appropriate.

In summary, this Special Issue contains a series of excellent research works on telecommunications and signal processing. This collection of 11 papers is highly recommended and believed to be interesting, inspiring, and motivating for researchers and other professionals.

Acknowledgments: We would like to thank all authors, the many dedicated referees, the editor team of Applied Sciences, and especially Xiaoyan Chen (Managing Editor) for their valuable contributions, making this special issue a success.

Conflicts of Interest: The authors declare no conflict of interest.

\section{References}

1. Martinez-Cruz, C.E.; Maier, G.; Pattavina, A.; Herencsar, N.; Tan, E. Global Communications Newsletter: The 2019 42nd International Conference on Telecommunications and Signal Processing (TSP) in Budapest, Hungary. IEEE Commun. Mag. 2019, 57, 8-11. doi:10.1109/MCOM.2019.8908543. [CrossRef]

2. Hilt, A. Availability and Fade Margin Calculations for 5G Microwave and Millimeter-Wave Anyhaul Links. Appl. Sci. 2019, 9, 5240. doi:10.3390/app9235240. [CrossRef]

3. Clupek, V.; Horvath, T.; Munster, P.; Oujezsky, V. New Security Improvements in Next-Generation Passive Optical Networks Stage 2 t. Appl. Sci. 2019, 9, 4430. doi:10.3390/app9204430. [CrossRef]

4. Boros, T.; Bencel, R.; Kotuliak, I. Transparent Redirections in Content Delivery Networks. Appl. Sci. 2019, 9 , 5418. doi:10.3390/app9245418. [CrossRef]

5. Sotner, R.; Domansky, O.; Jerabek, J.; Herencsar, N.; Petrzela, J.; Andriukaitis, D. Integer-and Fractional-Order Integral and Derivative Two-Port Summations: Practical Design Considerations. Appl. Sci. 2019, 10, 54. doi:10.3390/app10010054. [CrossRef]

6. Istvanek, M.; Smekal, Z.; Spurny, L.; Mekyska, J. Enhancement of Conventional Beat Tracking System Using Teager-Kaiser Energy Operator. Appl. Sci. 2020, 10, 379. doi:10.3390/app10010379. [CrossRef]

7. Ulacha, G.; Wernik, C. A High Efficiency Multistage Coder for Lossless Audio Compression using OLS+ and CDCCR Method. Appl. Sci. 2019, 9, 5218. doi:10.3390/app9235218. [CrossRef]

8. Datcu, O.; Macovei, C.; Hobincu, R. Chaos Based Cryptographic Pseudo-Random Number Generator Template with Dynamic State Change. Appl. Sci. 2020, 10, 451. doi:10.3390/app10020451. [CrossRef]

9. Romano, G. Asymptotic Performances of a Signal-To-Noise Ratio Moment-Based Estimator for Real Sinusoids in Additive Noise. Appl. Sci. 2019, 9, 5556. doi:10.3390/app9245556. [CrossRef] 
10. Riid, A.; Lõuk, R.; Pihlak, R.; Tepljakov, A.; Vassiljeva, K. Pavement Distress Detection with Deep Learning Using the Orthoframes Acquired by a Mobile Mapping System. Appl. Sci. 2019, 9, 4829. doi:10.3390/app9224829. [CrossRef]

11. Arjmand, A.; Angelis, C.T.; Christou, V.; Tzallas, A.T.; Tsipouras, M.G.; Glavas, E.; Forlano, R.; Manousou, P.; Giannakeas, N. Training of Deep Convolutional Neural Networks to Identify Critical Liver Alterations in Histopathology Image Samples. Appl. Sci. 2019, 10, 42. doi:10.3390/app10010042. [CrossRef]

12. Hanáková, L.; Průcha, J.; Socha, V.; Štengl, M.; Van den Bergh, S. Effect of High-Induction Magnetic Stimulation on Complex Heart Rate Variability of Sus Scrofa Domesticus under General Anesthesia. Appl. Sci. 2020, 10, 589. doi:10.3390/app10020589. [CrossRef]

2020 by the authors. Licensee MDPI, Basel, Switzerland. This article is an open access article distributed under the terms and conditions of the Creative Commons Attribution (CC BY) license (http://creativecommons.org/licenses/by/4.0/). 